\title{
Florística e estrutura do componente arbóreo de matas de Restinga arenosa no Parque Estadual de Itapuã, RS, Brasil
}

\author{
Adriano Scherer ${ }^{1,2}$, Fabiana Maraschin-Silva ${ }^{1}$ e Luís Rios de Moura Baptista ${ }^{1}$
}

Recebido em 18/10/2004. Aceito em 01/03/2005

\begin{abstract}
RESUMO - (Florística e estrutura do componente arbóreo de matas de Restinga arenosa no Parque Estadual de Itapuã, RS, Brasil). Para ampliar o conhecimento sobre as matas de Restinga arenosa no Rio Grande do Sul, foi realizado o estudo florístico e fitossociológico do componente arbóreo de cinco capões de Restinga do Parque Estadual de Itapuã. Neste estudo comparativo foi usado o método de parcelas, incluindo todos os indivíduos com DAP mínimo de $5 \mathrm{~cm}$ e totalizando uma área de 1,02 ha. A composição florística resultou em uma riqueza total de 31 espécies, 26 gêneros e 20 famílias, considerando os cinco capões juntos. A densidade total arbórea nos capões teve uma média de 1.023 ind/ha. A família com a maior riqueza de espécies foi Myrtaceae e, entre as espécies com maior valor de importância, foram encontradas Sebastiania serrata (Baill. ex Müll. Arg.) Müll. Arg. e Ficus organensis Miq. A diversidade específica foi baixa, variando de 1,08 a 2,38 nats.ind ${ }^{-1}$. Os resultados obtidos indicam a existência de diferenças na composição e na estrutura arbórea dos capões que podem ser oriundas de diferentes estágios sucessionais e da distância de áreas fontes de sementes.
\end{abstract}

Palavras-chave: Restinga, diversidade, fitossociologia, componente arbóreo, Itapuã

\begin{abstract}
Floristic and structure of the tree component of sandy Restinga forests in Parque Estadual de Itapuã, Rio Grande do Sul State, Brazil). To improve the knowledge about sandy Restinga forests in Rio Grande do Sul, the floristic and phytosociological study of the tree component of five Restinga woods from Parque Estadual de Itapuã was accomplished. In this comparative study, the plot method was used, including all individuals with minimum DBH of $5 \mathrm{~cm}$ and totaling an area of 1,02 ha. The floristic composition resulted in a total richness of 31 species, 26 genera and 20 families, considering the five woods together. The total tree density in the woods had a media of 1,023 ind/ha. The richest family was Myrtaceae and, among the most important species, Sebastiania serrata (Baill. ex Müll. Arg.) Müll. Arg. and Ficus organensis Miq. were found. Species diversity was low, varying from 1,08 to 2,38 nats.ind ${ }^{-1}$. The results obtained point to the existence of differences in the forests tree composition and structure that may outcome from different sucessional stages and from the seed source area distance.
\end{abstract}

Key words: Restinga, diversity, phytosociology, tree component, Itapuã

\section{Introdução}

A denominação Restinga, embora amplamente utilizada, não apresenta uma uniformidade no sentido geológico, sendo normalmente usada para denominar qualquer depósito arenoso litorâneo brasileiro (Falkenberg 1999). Em um contexto ecológico, as Restingas englobam todas as comunidades vegetais e animais do litoral arenoso e seus ambientes físicos (Waechter 1985). Considerando a definição de Restinga para o sul do Brasil, este ecossistema se estende da porção mais austral do Estado de Santa Catarina até o extremo sul do Rio Grande do Sul (Waechter 1985; Araújo 1992), formando um conjunto de ambientes costeiros normalmente agregados às lagoas litorâneas e apresentando comunidades vegetais com fisionomias distintas (Rambo 1956).

A vegetação nesta região recobre em maior parte depósitos eólicos, representados por dunas fixas ou móveis (Teixeira et al. 1986). Essas áreas caracterizam-se por apresentar formação sedimentar do período quaternário, com raros afloramentos de rochas antigas, como o granito pré-cambriano de Itapuã e São Lourenço do Sul, o arenito triássico e o basalto jurássico de Torres e Itapeva (Waechter 1985). As comunidades vegetais da Restinga sul-brasileira constituem um mosaico de acordo com as características edáficas, que resultam de pequenas

1 Universidade Federal do Rio Grande do Sul, Programa de Pós-Graduação em Botânica, Av. Bento Gonçalves 9500, Bloco 4, Prédio 43433, Sala 214, CEP 91509-900, Porto Alegre, RS, Brasil

2 Autor para correspondência: adriano@sisrest.org.br 
variações topográficas, da natureza e idade dos diferentes depósitos geológicos, associadas às condições de clima úmido (S.C. Müller, dados não publicados).

De modo geral, as Restingas apresentam espécies arbóreas provenientes de outros ecossistemas, que colonizaram estes ambientes em razão da variedade das condições físicas que ali ocorrem (Rambo 1954; Araújo \& Lacerda 1987; Freire 1990), porém apresentam variações fisionômicas devido às condições distintas do seu ambiente de origem (Assumpção \& Nascimento 2000). Nestas formações há significativa diminuição da diversidade específica no sentido norte-sul, fato que termina por influenciar sensivelmente o aspecto fisionômico. Esta diferenciação na flora começa a ocorrer na altura do paralelo $30^{\circ}$, que pode ser considerado uma fronteira mais avançada de ocorrência da vegetação tipicamente tropical na costa oriental da América do Sul (Waechter 1985).

Neste âmbito, as comunidades arbóreas de substrato arenoso, também conhecidas regionalmente como capões de Restinga (Lindman 1906; Waechter 1985), ocorrem praticamente ao longo de toda a região geomorfológica denominada Planície Costeira do Rio Grande do Sul (Waechter 1990). Nas áreas mais internas desta região, estas formações vegetais ocorrem predominantemente sobre depósitos planos ou suavemente ondulados, apresentando porte geralmente mais desenvolvido, diferentemente daquelas sobre barreiras quartzosas mais próximas do oceano (Waechter 1990).

Estas matas de Restinga arenosa apresentam certa semelhança fisionômica e florística com as matas rupestres, como as que ocorrem nos morros graníticos de Porto Alegre e arredores (Waechter 1985), sobressaindo-se as da zona sul da cidade. Nesta região, existem matas psamófilas que apresentam características xeromórficas (Brack et al. 1998).

$\mathrm{O}$ fato de serem áreas de formações vegetais sobre solos recentes, tais como planossolos e neossolos (Teixeira et al. 1986; Streck et al. 2002), revela um caráter especial nestes ecossistemas. A vegetação exerce papel fundamental para a estabilização do substrato nestes ambientes, protegendo da ação do vento, que se constitui em importante agente modificador da paisagem (Lindman 1906; Lamego 1946; Rambo 1956; Costa et al. 1984; Assumpção e Nascimento 2000), e mantendo a drenagem natural, bem como preservando a fauna residente e migratória (Falkenberg 1999). Mesmo apresentando tal importância, os ecossistemas de Restinga têm sofrido crescentes impactos nos últimos 50 anos, principalmente devido à especulação imobiliária, invasão de espécies exóticas e expansão das áreas de agropecuária.

Diversos trabalhos sobre as comunidades de Restinga têm sido feitos no Rio Grande do Sul ao longo dos anos, sendo contemplados somente aspectos sobre a composição florística, em sua maioria. São poucos, contudo, os estudos que abordam a fitossociologia das matas de Restinga arenosa, destacando-se os de Dillenburg et al. (1992), Rossoni \& Baptista (1994; 1995), Waechter et al. (2000), Moraes \& Mondin (2001) e Dorneles \& Waechter (2004).

Visando ampliar o conhecimento destas formações vegetais, o presente estudo tem como objetivo analisar a composição florística e a estrutura fitossociológica do componente arbóreo de um cordão de capões de Restinga arenosa da Praia de Fora no Parque Estadual de Itapuã.

\section{Material e métodos}

Área de Estudo - O Parque Estadual de Itapuã encontra-se na região de divisa entre a Depressão Central e a Planície Costeira do Rio Grande do Sul, sendo banhado pelo lago Guaíba a oeste e pela lagoa dos Patos ao sul e leste. O parque possui uma área total de 5.566,50 ha, sediado no município de Viamão, distante cerca de $57 \mathrm{~km}$ do centro de Porto Alegre.

A Praia de Fora é a maior praia da unidade de conservação, com $16 \mathrm{~km}$ de extensão, iniciando junto ao morro de Itapuã e se estendendo até o Pontal das Desertas. A região da Praia de Fora, onde se localiza a área de estudo, apresenta solo arenoso, composto por areias quartzosas não fixadas, de granulação fina e média, bem drenado, formando extensos campos de dunas com altitudes de 10 a $25 \mathrm{~m}$. A vegetação local é constituída por campos arenosos e alagados, formações arbustivas (moitas) e capões de Restinga arenosa. Além disto, nessa região encontra-se uma lagoa com 1.750 ha, a Lagoa Negra, que corresponde a aproximadamente $31 \%$ da área total do Parque (Rio Grande do Sul 1997).

Anteriormente à implantação do Parque Estadual de Itapuã, a região da Praia de Fora sofreu grande pressão antrópica, tendo existido no local aproximadamente 800 casas construídas irregularmente por invasores (Rio Grande do Sul 1997). Com a efetividade da unidade de conservação em 1991, as casas e pessoas foram retiradas e desde então o acesso à região da Praia de Fora ficou restrito aos pesquisadores e guardasparque. 
Segundo o sistema de Köppen, o clima da região onde se localiza o Parque é classificado como Cfa, subtropical úmido, podendo haver estiagem, com a temperatura do mês mais quente ultrapassando $22^{\circ} \mathrm{C}$ e a do mês mais frio inferior a $18{ }^{\circ} \mathrm{C}$ e superior a $3{ }^{\circ} \mathrm{C}$, com médias mensais compreendidas entre 10 e $22^{\circ} \mathrm{C}$, com ocorrência de geadas (Mota 1951). A pluviosidade e a temperatura média anual são de $1.300 \mathrm{~mm}$ e $17,5^{\circ} \mathrm{C}$ respectivamente. $\mathrm{O}$ vento predominante, assim como em toda Planície Costeira, é o nordeste (Rio Grande do Sul 1997).

O estudo da vegetação arbórea foi feito em cinco capões de Restinga contíguos, localizados às margens da Lagoa Negra, que foram denominados respectivamente: 1) Mato da Tapera, $30^{\circ} 22^{\prime} 47,7^{\prime \prime} \mathrm{S}$ $51^{\circ} 00^{\prime} 40,9^{\prime \prime} \mathrm{W}$; 2) Mato da Lagoinha, 30²2'48,9”S

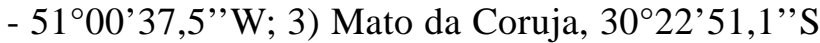
- 51 ${ }^{\circ} 00^{\prime} 29,0^{\prime \prime} \mathrm{W}$; 4) Mato do Puma, 30²2'51,9''S $51^{\circ} 00^{\prime} 15,3^{\prime \prime} \mathrm{W}$ e 5) Mato das Capivaras 30²3'15,5"S - 5058'43,9”W. Na área dos capões, parte da vegetação arbórea foi retirada seletivamente para construção de casas durante a ocupação dos invasores. Próximos aos capões há um morro granítico, $30^{\circ} 22^{\prime} 47,7$ 'S - $51^{\circ} 00^{\prime} 06,3$ 'W, tendo as seguintes distâncias dos capões: $650 \mathrm{~m}$ para o da Tapera, $720 \mathrm{~m}$ para o da Lagoinha, $950 \mathrm{~m}$ para o da Coruja, $1.300 \mathrm{~m}$ para o do Puma e $3.850 \mathrm{~m}$ para o das Capivaras. Esse morro apresenta uma cobertura vegetal formada por Floresta Estacional Semidecidual (Knob 1975; Aguiar et al. 1986; Backes 1999; P. Oliveira-Neves, dados não publicados), constituindo-se em uma provável matriz de espécies que ocorrem nesses capões.

Procedimento amostral - No levantamento fitossociológico, foi empregado o método de parcelas, com $10 \times 10 \mathrm{~m}$ de tamanho (Mueller-Dombois \& Ellenberg 1974), procurando-se cobrir cada capão com o máximo de parcelas possível. Foram levantadas uma área de 0,13 ha para o Mato da Tapera, 0,2 ha para o Mato da Lagoinha, 0,29 ha para o Mato da Coruja, 0,26 ha para o Mato do Puma e 0,14 ha para o Mato das Capivaras, totalizando 1,02 ha. Como critério de inclusão para as árvores, foi usado DAP $(1,30 \mathrm{~m})$ $\geq 5 \mathrm{~cm}$. Aqueles indivíduos que se encontravam sobre as bordas da parcelas foram considerados, desde que ao menos metade de seus diâmetros estivesse no interior da unidade amostral. Além da medida do DAP, a altura total das árvores foi estimada visualmente, comparando-se com uma estaca de tamanho conhecido. No levantamento fitossociológico, não foram amostradas as espécies da família Cactaceae.
A identificação das espécies foi feita no local ou em análise posterior, utilizando-se a bibliografia disponível, fotografias e comparações com exsicatas de herbário, conforme a necessidade. Foram feitas exsicatas das espécies levantadas, estando estas depositadas no Herbário ICN do Departamento de Botânica da Universidade Federal do Rio Grande do Sul. As famílias foram classificadas de acordo com APGII (2003) e a atualização nomenclatural da família Myrtaceae foi feita de acordo com Sobral (2003).

Análise de dados - A estratificação dos capões foi analisada através de um diagrama de alturas. Também foram estimados os parâmetros de densidade, freqüência e cobertura, absolutas e relativas, e índice de valor de importância (Mueller-Dombois \& Ellenberg 1974). Além destes parâmetros, foram avaliados: a riqueza, pelo número de espécies $(\mathrm{S})$ e pelo índice $\mathrm{R}$ de Gleasson (1922), que permite comparações independentes do tamanho da área; a diversidade específica, pelo índice H' de Shannon; a equabilidade, pelo índice E de Pielou; e a concentração, pelo índice C de Simpson (Kent \& Coker 1995; Durigan 2003). Para comparação da similaridade florística foi usado o índice de Jaccard (Kent \& Coker 1995; Durigan 2003), sendo que para comparações com outras áreas de mata de Restinga já estudadas foi considerado o total de espécies arbóreas inventariadas nos cinco capões. Com os dados obtidos foram elaboradas matrizes de presença e ausência das espécies (composição florística) e/ou com valor de importância, nas quais cada mata foi considerada como uma unidade amostral. A partir destas matrizes foram feitas: medidas de semelhança, através do Índice de Jaccard; análise de agrupamento, usando-se como medida de similaridade o Índice de Jaccard e ligação média não-ponderada como critério de agrupamento das unidades amostrais; e análise de ordenação, através de Análise de Coordenadas Principais. Para estas análises, foi utilizado o programa Multiv (Pillar 2001).

\section{Resultados}

Considerando-se todos os capões de Restinga estudados, obteve-se uma riqueza de 31 espécies, distribuídas em 26 gêneros, pertencentes a 20 famílias (Tab. 1). Em comparação com o levantamento fitossociológico, a composição florística incluiu também duas espécies da família Cactaceae. A família com maior riqueza específica foi Myrtaceae, que somou um total de sete espécies, sendo que quatro destas 
Tabela 1. Composição florística dos capões de Restinga do Parque Estadual de Itapuã, RS, Brasil.

\begin{tabular}{|c|c|}
\hline Família/Espécie & Nome popular \\
\hline \multicolumn{2}{|l|}{ ANACARDIACEAE } \\
\hline Lithraea brasiliensis Marchand & aroeira-brava \\
\hline \multicolumn{2}{|l|}{ ANNONACEAE } \\
\hline Rollinia maritima Schltdl. & araticum \\
\hline \multicolumn{2}{|l|}{ ARECACEAE } \\
\hline \multicolumn{2}{|l|}{ Glassman } \\
\hline \multicolumn{2}{|l|}{ BIGNONIACEAE } \\
\hline Tabebuia pulcherrima Sandwith & ipê \\
\hline \multicolumn{2}{|l|}{ CACTACEAE } \\
\hline Cereus hildmannianus K. Schum. & tuna \\
\hline Opuntia monacantha (Willd.) Haw. & palmatória \\
\hline \multicolumn{2}{|l|}{ ERYTHROXYLACEAE } \\
\hline Erythroxylum argentinum O.E. Schulz & cocão \\
\hline \multicolumn{2}{|l|}{ EUPHORBIACEAE } \\
\hline $\begin{array}{l}\text { Sebastiania serrata (Baill. ex Müll. } \\
\text { Arg.) Müll. Arg. }\end{array}$ & branquilho \\
\hline \multicolumn{2}{|l|}{ LAMIACEAE } \\
\hline Vitex megapotamica (Spreng.) Moldenke & tarumã \\
\hline \multicolumn{2}{|l|}{ LAURACEAE } \\
\hline Ocotea pulchella Mart. & canela-lageana \\
\hline \multicolumn{2}{|l|}{ MORACEAE } \\
\hline Ficus organensis Miq. & figueira \\
\hline \multicolumn{2}{|l|}{ MYRSINACEAE } \\
\hline Myrsine guianensis (Aubl.) Kuntze & capororoca \\
\hline Myrsine umbellata Mart. & capororoca \\
\hline \multicolumn{2}{|l|}{ MYRTACEAE } \\
\hline Eugenia hiemalis Cambess. & guamirim-burro \\
\hline Eugenia myrcianthes Nied. & pessegueiro-do-mato \\
\hline Eugenia speciosa Cambess. & araçá \\
\hline Eugenia uruguayensis Cambess. & guamirim \\
\hline Myrcia palustris DC. & guamirim \\
\hline Myrciaria cuspidata $\mathrm{O}$. Berg & camboim \\
\hline Psidium cattleyanum Sabine & araçá \\
\hline \multicolumn{2}{|l|}{ NYCTAGINACEAE } \\
\hline Guapira opposita (Vell.) Reitz & maria-mole \\
\hline \multicolumn{2}{|l|}{ RUBIACEAE } \\
\hline Randia armata (Sw.) DC. & limoeiro-do-mato \\
\hline \multicolumn{2}{|l|}{ RUTACEAE } \\
\hline Zanthoxylum fagara (L.) Sarg. & mamica-de-cadela \\
\hline \multicolumn{2}{|l|}{ SALICACEAE } \\
\hline Casearia decandra Jacq. & guaçatunga \\
\hline Casearia sylvestris Sw. & chá-de-bugre \\
\hline \multicolumn{2}{|l|}{ SAPINDACEAE } \\
\hline Dodonaea viscosa Jacq. & vassoura-vermelha \\
\hline \multicolumn{2}{|l|}{ SAPOTACEAE } \\
\hline $\begin{array}{l}\text { Chrysophyllum marginatum (Hook. } \\
\text { \& Arn.) Radlk. }\end{array}$ & aguaí \\
\hline $\begin{array}{l}\text { Sideroxylon obtusifolium (Humb. ex } \\
\text { Roem. \& Schult.) T.D. Penn. }\end{array}$ & coronilha \\
\hline \multicolumn{2}{|l|}{ SOLANACEAE } \\
\hline Solanum pseudoquina A. St.-Hil. & canema \\
\hline \multicolumn{2}{|l|}{ URTICACEAE } \\
\hline Cecropia pachystachya Trécul & embaúba \\
\hline Coussapoa microcarpa (Schott) Rizzini & mata-pau \\
\hline
\end{tabular}

pertencem ao gênero Eugenia. As famílias Cactaceae, Urticaceae, Salicaceae, Myrsinaceae e Sapotaceae apresentaram duas espécies e as demais tiveram apenas uma.

Foi estimada uma densidade total média de $1.023 \mathrm{ind} / \mathrm{ha}$ para os capões estudados. Entretanto, constatou-se que, nos três capões mais próximos ao morro, a densidade total foi superior $1.000 \mathrm{ind} / \mathrm{ha}$, sendo que nos demais esta densidade cai para valores abaixo de 800 ind/ha (Tab. 2).

As cinco espécies mais importantes nos capões somaram aproximadamente 50,60, 75, 65 e $90 \%$ do Índice de Valor de Importância (IVI) respectivamente para: Mato da Tapera, Mato da Lagoinha, Mato da Coruja, Mato do Puma e Mato das Capivaras (Tab. 2). As estimativas dos parâmetros fitossociológicos demonstraram a elevada importância de Sebastiania serrata em todos os capões, com maior contribuição da densidade e freqüência relativas (DR e FR). Ficus organensis e Sideroxylon obtusifolium apresentaram elevados valores de cobertura relativa (CR), em função do diâmetro relativamente grosso dos troncos.

A riqueza foi maior no Mato da Lagoinha, considerando-se tanto o número de espécies (S) como o Índice de Gleasson (Tab. 3). Os matos da Tapera, da Lagoinha e do Puma apresentaram valores mais altos para diversidade e equidade. Já os matos da Coruja e das Capivaras tiveram maior concentração.

Os matos da Tapera, Lagoinha e Coruja mostraram maior similaridade florística entre si, com aproximadamente $60 \%$ de semelhança, conforme demonstrado na análise de agrupamento (Fig. 1). Os menores valores para o Índice de Similaridade de Jaccard foram encontrados entre o Mato da Lagoinha e o Mato das Capivaras, bem como entre o Mato da Tapera e o do Puma.

Em relação a outras áreas, a maior semelhança na composição florística foi com a mata psamófila do Arroio Itapuã, apresentando uma similaridade de $45 \%$ (Tab. 4). A mata de Restinga de Rondinha Velha, por sua vez, teve menor similaridade com os capões de Restinga do Parque Estadual de Itapuã (27,14\%). A diversidade estimada pelo índice de Shannon foi próxima aos valores encontrados em outras áreas (Tab. 3 e Tab. 4).

As alturas estimadas para as árvores variaram entre 2 a $15 \mathrm{~m}$, considerando-se todos os capões (Fig. 2). Ficaram evidenciados um estrato superior, em torno de $4 \mathrm{~m}$ para os matos das Capivaras e do Puma e de 5 a 6 m para os demais; e um grupo de 
Tabela 2. Parâmetros fitossociológicos do componente arbóreo dos capões de Restinga do Parque Estadual de Itapuã, RS, Brasil. DA: densidade absoluta (ind/ha), DR: densidade relativa (\%), FA: freqüência absoluta (\%), FR: freqüência relativa(\%), CA: cobertura absoluta $\left(\mathrm{m}^{2}\right)$, CR: cobertura relativa (\%), IVI: índice do valor de importância (\%), IVC: índice do valor de cobertura (\%).

\begin{tabular}{|c|c|c|c|c|c|c|c|c|}
\hline Espécie & DA & DR & FA & FR & $\mathrm{CA}$ & $\mathrm{CR}$ & IVI & IVC \\
\hline \multicolumn{9}{|l|}{ Mato da Tapera } \\
\hline Sebastiania serrata & 285 & 24,67 & 92,31 & 15,38 & 0,756 & 18,661 & 19,57 & 21,66 \\
\hline Tabebuia pulcherrima & 154 & 13,33 & 53,85 & 8,97 & 0,354 & 8,729 & 10,35 & 11,03 \\
\hline Ficus organensis & 15 & 1,33 & 15,38 & 2,56 & 1,078 & 26,595 & 10,16 & 13,96 \\
\hline Ocotea pulchella & 123 & 10,67 & 61,54 & 10,26 & 0,351 & 8,655 & 9,86 & 9,66 \\
\hline Eugenia uruguayensis & 131 & 11,33 & 61,54 & 10,26 & 0,184 & 4,530 & 8,71 & 7,93 \\
\hline Myrciaria cuspidata & 100 & 8,67 & 76,92 & 12,82 & 0,168 & 4,142 & 8,54 & 6,40 \\
\hline Casearia sylvestris & 77 & 6,67 & 38,46 & 6,41 & 0,345 & 8,503 & 7,19 & 7,58 \\
\hline Myrsine guianensis & 62 & 5,33 & 46,15 & 7,69 & 0,256 & 6,308 & 6,44 & 5,82 \\
\hline Myrcia palustris & 46 & 4,00 & 38,46 & 6,41 & 0,081 & 2,010 & 4,14 & 3,00 \\
\hline Zanthoxylum fagara & 38 & 3,33 & 30,77 & 5,13 & 0,051 & 1,268 & 3,24 & 2,30 \\
\hline Sideroxylon obtusifolium & 8 & 0,67 & 7,69 & 1,28 & 0,299 & 7,390 & 3,11 & 4,03 \\
\hline Vitex megapotamica & 23 & 2,00 & 23,08 & 3,85 & 0,025 & 0,627 & 2,16 & 1,31 \\
\hline Eugenia speciosa & 31 & 2,67 & 15,38 & 2,56 & 0,015 & 0,369 & 1,87 & 1,52 \\
\hline Chrysophyllum marginatum & 23 & 2,00 & 15,38 & 2,56 & 0,037 & 0,915 & 1,83 & 1,46 \\
\hline Casearia decandra & 15 & 1,33 & 7,69 & 1,28 & 0,022 & 0,538 & 1,05 & 0,94 \\
\hline Eugenia hiemalis & 15 & 1,33 & 7,69 & 1,28 & 0,016 & 0,397 & 1,00 & 0,86 \\
\hline \multirow[t]{2}{*}{ Eugenia myrcianthes } & 8 & 0,67 & 7,69 & 1,28 & 0,015 & 0,363 & 0,77 & 0,51 \\
\hline & 1.154 & 100,00 & 600,00 & 100,00 & 4,05 & 100,000 & 100,00 & 100,00 \\
\hline \multicolumn{9}{|l|}{ Mato da Lagoinha } \\
\hline Sebastiania serrata & 525 & 37,91 & 90 & 14,88 & 2,15 & 31,85 & 28,21 & 34,88 \\
\hline Guapira opposita & 155 & 11,19 & 40 & 6,61 & 0,64 & 9,46 & 9,09 & 10,33 \\
\hline Vitex megapotamica & 50 & 3,61 & 50 & 8,26 & 0,93 & 13,77 & 8,55 & 8,69 \\
\hline Ficus organensis & 15 & 1,08 & 15 & 2,48 & 1,36 & 20,14 & 7,90 & 10,61 \\
\hline Tabebuia pulcherrima & 135 & 9,75 & 45 & 7,44 & 0,26 & 3,81 & 7,00 & 6,78 \\
\hline Myrciaria cuspidata & 110 & 7,94 & 60 & 9,92 & 0,18 & 2,59 & 6,82 & 5,27 \\
\hline Casearia sylvestris & 65 & 4,69 & 45 & 7,44 & 0,29 & 4,32 & 5,48 & 4,51 \\
\hline Zanthoxylum fagara & 40 & 2,89 & 30 & 4,96 & 0,08 & 1,12 & 2,99 & 2,00 \\
\hline Ocotea pulchella & 40 & 2,89 & 30 & 4,96 & 0,07 & 1,10 & 2,98 & 1,99 \\
\hline Erythroxylum argentinum & 35 & 2,53 & 25 & 4,13 & 0,10 & 1,54 & 2,73 & 2,03 \\
\hline Solanum pseudoquina & 30 & 2,17 & 30 & 4,96 & 0,03 & 0,46 & 2,53 & 1,31 \\
\hline Eugenia uruguayensis & 35 & 2,53 & 20 & 3,31 & 0,09 & 1,30 & 2,38 & 1,91 \\
\hline Myrsine guianensis & 20 & 1,44 & 20 & 3,31 & 0,12 & 1,84 & 2,20 & 1,64 \\
\hline Myrcia palustris & 25 & 1,81 & 20 & 3,31 & 0,04 & 0,57 & 1,89 & 1,19 \\
\hline Chrysophyllum marginatum & 15 & 1,08 & 15 & 2,48 & 0,10 & 1,46 & 1,67 & 1,27 \\
\hline Dodonaea viscosa & 20 & 1,44 & 15 & 2,48 & 0,04 & 0,59 & 1,50 & 1,02 \\
\hline Casearia decandra & 25 & 1,81 & 10 & 1,65 & 0,04 & 0,56 & 1,34 & 1,18 \\
\hline Syagrus romanzoffiana & 10 & 0,72 & 10 & 1,65 & 0,06 & 0,92 & 1,10 & 0,82 \\
\hline Sideroxylon obtusifolium & 5 & 0,36 & 5 & 0,83 & 0,14 & 2,08 & 1,09 & 1,22 \\
\hline Eugenia hiemalis & 10 & 0,72 & 10 & 1,65 & 0,01 & 0,14 & 0,84 & 0,43 \\
\hline Randia armata & 10 & 0,72 & 10 & 1,65 & 0,01 & 0,14 & 0,84 & 0,43 \\
\hline Lithraea brasiliensis & 5 & 0,36 & 5 & 0,83 & 0,01 & 0,20 & 0,46 & 0,28 \\
\hline \multirow[t]{2}{*}{ Rollinia maritima } & 5 & 0,36 & 5 & 0,83 & 0,00 & 0,03 & 0,40 & 0,19 \\
\hline & 1.385 & 100,00 & 605 & 100,00 & 6,76 & 100,00 & 100,00 & 100,00 \\
\hline \multicolumn{9}{|l|}{ Mato da Coruja } \\
\hline Sebastiania serrata & 414 & 38,22 & 89,66 & 18,84 & 2,335 & 30,20 & 29,09 & 34,21 \\
\hline Myrcia palustris & 176 & 16,24 & 72,41 & 15,22 & 1,041 & 13,47 & 14,98 & 14,86 \\
\hline Myrsine guianensis & 152 & 14,01 & 82,76 & 17,39 & 0,676 & 8,74 & 13,38 & 11,38 \\
\hline Vitex megapotamica & 72 & 6,69 & 58,62 & 12,32 & 0,821 & 10,62 & 9,88 & 8,65 \\
\hline Ficus organensis & 10 & 0,96 & 10,34 & 2,17 & 1,803 & 23,32 & 8,82 & 12,14 \\
\hline Myrciaria cuspidata & 76 & 7,01 & 41,38 & 8,70 & 0,288 & 3,73 & 6,48 & 5,37 \\
\hline Ocotea pulchella & 62 & 5,73 & 31,03 & 6,52 & 0,281 & 3,64 & 5,30 & 4,69 \\
\hline Eugenia uruguayensis & 55 & 5,10 & 37,93 & 7,97 & 0,108 & 1,40 & 4,82 & $\begin{array}{l}3,25 \\
\text { cont }\end{array}$ \\
\hline
\end{tabular}


Tabela 2 (continuação)

\begin{tabular}{|c|c|c|c|c|c|c|c|c|}
\hline Espécie & $\mathrm{DA}$ & DR & FA & FR & $\mathrm{CA}$ & CR & IVI & IVC \\
\hline Solanum pseudoquina & 10 & 0,96 & 10,34 & 2,17 & 0,113 & 1,46 & 1,53 & 1,21 \\
\hline Eugenia myrcianthes & 14 & 1,27 & 10,34 & 2,17 & 0,061 & 0,79 & 1,41 & 1,03 \\
\hline Randia armata & 14 & 1,27 & 6,90 & 1,45 & 0,061 & 0,79 & 1,17 & 1,03 \\
\hline Zanthoxylum fagara & 7 & 0,64 & 6,90 & 1,45 & 0,077 & 1,00 & 1,03 & 0,82 \\
\hline Chrysophyllum marginatum & 7 & 0,64 & 3,45 & 0,72 & 0,042 & 0,54 & 0,63 & 0,59 \\
\hline Casearia sylvestris & 3 & 0,32 & 3,45 & 0,72 & 0,012 & 0,16 & 0,40 & 0,24 \\
\hline Dodonaea viscosa & 3 & 0,32 & 3,45 & 0,72 & 0,006 & 0,07 & 0,37 & 0,20 \\
\hline Sideroxylon obtusifolium & 3 & 0,32 & 3,45 & 0,72 & 0,003 & 0,04 & 0,36 & 0,18 \\
\hline \multirow[t]{2}{*}{ Myrsine umbellata } & 3 & 0,32 & 3,45 & 0,72 & 0,002 & 0,02 & 0,36 & 0,17 \\
\hline & 1.083 & 100,00 & 476,00 & 100,00 & 7,730 & 100,00 & 100,00 & 100,00 \\
\hline \multicolumn{9}{|l|}{ Mato do Puma } \\
\hline Sebastiania serrata & 200 & 28,57 & 73,08 & 20,21 & 1,361 & 19,40 & 22,73 & 23,99 \\
\hline Dodonaea viscosa & 188 & 26,92 & 73,08 & 20,21 & 0,276 & 3,93 & 17,02 & 15,43 \\
\hline Guapira opposita & 38 & 5,49 & 26,92 & 7,45 & 1,557 & 22,20 & 11,71 & 13,85 \\
\hline Sideroxylon obtusifolium & 19 & 2,75 & 19,23 & 5,32 & 0,852 & 12,15 & 6,74 & 7,45 \\
\hline Myrsine guianensis & 35 & 4,95 & 26,92 & 7,45 & 0,352 & 5,02 & 5,80 & 4,98 \\
\hline Eugenia myrcianthes & 35 & 4,95 & 30,77 & 8,51 & 0,098 & 1,40 & 4,95 & 3,17 \\
\hline Eugenia uruguayensis & 58 & 8,24 & 15,38 & 4,26 & 0,141 & 2,02 & 4,84 & 5,13 \\
\hline Ficus organensis & 4 & 0,55 & 3,85 & 1,06 & 0,817 & 11,65 & 4,42 & 6,10 \\
\hline Solanum pseudoquina & 27 & 3,85 & 23,08 & 6,38 & 0,117 & 1,66 & 3,96 & 2,75 \\
\hline Coussapoa microcarpa & 8 & 1,10 & 7,69 & 2,13 & 0,599 & 8,54 & 3,92 & 4,82 \\
\hline Vitex megapotamica & 11 & 1,65 & 11,54 & 3,19 & 0,296 & 4,22 & 3,02 & 2,93 \\
\hline Ocotea pulchella & 15 & 2,20 & 7,69 & 2,13 & 0,237 & 3,38 & 2,57 & 2,79 \\
\hline Syagrus romanzoffiana & 15 & 2,20 & 3,85 & 1,06 & 0,176 & 2,51 & 1,92 & 2,35 \\
\hline Cecropia pachystachya & 15 & 2,20 & 7,69 & 2,13 & 0,059 & 0,85 & 1,72 & 1,52 \\
\hline Myrsine umbellata & 11 & 1,65 & 11,54 & 3,19 & 0,021 & 0,30 & 1,71 & 0,97 \\
\hline Psidium cattleyanum & 8 & 1,10 & 7,69 & 2,13 & 0,025 & 0,36 & 1,20 & 0,73 \\
\hline Erythroxylum argentinum & 8 & 1,10 & 7,69 & 2,13 & 0,025 & 0,35 & 1,19 & 0,73 \\
\hline \multirow[t]{2}{*}{ Myrcia palustris } & 4 & 0,55 & 3,85 & 1,06 & 0,005 & 0,07 & 0,56 & 0,31 \\
\hline & 700 & 100,00 & 361,00 & 100,00 & 9,680 & 100,00 & 100,00 & 100,00 \\
\hline \multicolumn{9}{|l|}{ Mato das Capivaras } \\
\hline Sebastiania serrata & 579 & 72,97 & 100,00 & 40,00 & 1,870 & 60,44 & 57,80 & 66,71 \\
\hline Dodonaea viscosa & 64 & 8,11 & 42,86 & 17,14 & 0,040 & 1,29 & 8,85 & 4,70 \\
\hline Eugenia myrcianthes & 57 & 7,21 & 35,71 & 14,29 & 0,116 & 3,75 & 8,42 & 5,48 \\
\hline Ficus organensis & 7 & 0,90 & 7,14 & 2,86 & 0,584 & 18,89 & 7,55 & 9,90 \\
\hline Vitex megapotamica & 14 & 1,80 & 14,29 & 5,71 & 0,363 & 11,75 & 6,42 & 6,77 \\
\hline Myrsine guianensis & 29 & 3,60 & 14,29 & 5,71 & 0,070 & 2,27 & 3,86 & 2,94 \\
\hline Sideroxylon obtusifolium & 21 & 2,70 & 14,29 & 5,71 & 0,021 & 0,68 & 3,03 & 1,69 \\
\hline Myrcia palustris & 7 & 0,90 & 7,14 & 2,86 & 0,018 & 0,57 & 1,44 & 0,73 \\
\hline Myrciaria cuspidata & 7 & 0,90 & 7,14 & 2,86 & 0,006 & 0,21 & 1,32 & 0,55 \\
\hline \multirow[t]{2}{*}{ Myrsine umbellata } & 7 & 0,90 & 7,14 & 2,86 & 0,005 & 0,15 & 1,30 & 0,52 \\
\hline & 793 & 100,00 & 250,00 & 100,00 & 3,090 & 100,00 & 100,00 & 100,00 \\
\hline
\end{tabular}

emergentes, com 9 a $15 \mathrm{~m}$ para todos os capões, tais como Coussapoa microcarpa, Ficus organensis, Syagrus romanzoffiana e Tabebuia pulcherrima. Constatou-se também uma grande porcentagem de indivíduos com alturas mais baixas ( 2 a $4 \mathrm{~m}) \mathrm{e}$ intermediárias (5 a $8 \mathrm{~m}$ para Capivaras e Puma e 6 a $8 \mathrm{~m}$ para os demais capões), não sendo, contudo, visualizados picos modais no diagrama de alturas (Fig. 2).

$\mathrm{Na}$ ordenação usando a composição florística, representada pelo diagrama da Fig. 3a, o Eixo I teve
Tabela 3. Medidas de diversidade nos capões de Restinga do Parque Estadual de Itapuã, RS, Brasil. S = Riqueza (número de espécies); $\mathrm{R}=$ Riqueza (Índice de Gleasson), $\mathrm{H}^{\prime}=$ diversidade (nats.ind ${ }^{-1}$ ), $\mathrm{E}=$ equabilidade, $\mathrm{C}=$ concentração.

\begin{tabular}{lccccc}
\hline Capões & S & R & H' & E & C \\
\hline Mato da Tapera & 17 & 3,49 & 2,38 & 0,841 & 0,122 \\
Mato da Lagoinha & 23 & 4,34 & 2,31 & 0,737 & 0,180 \\
Mato da Coruja & 17 & 3,00 & 1,96 & 0,691 & 0,208 \\
Mato do Puma & 18 & 3,23 & 2,19 & 0,758 & 0,173 \\
Mato das Capivaras & 10 & 2,02 & 1,08 & 0,470 & 0,547 \\
\hline
\end{tabular}


Índice de similaridade de Jaccard

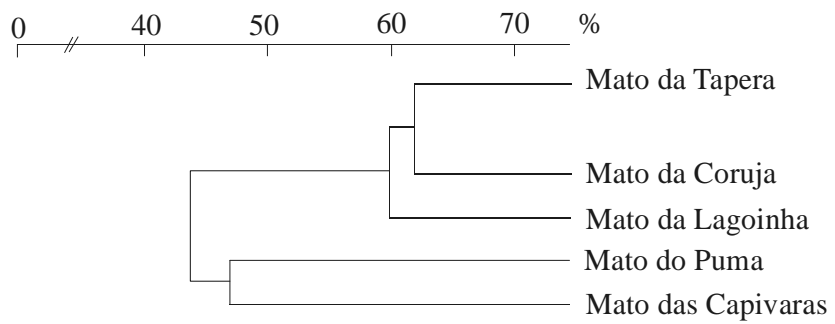

Figura 1. Análise de agrupamento dos cinco capões de Restinga do Parque Estadual de Itapuã, RS, Brasil.

Tabela 4. Comparação da composição florística e da diversidade dos capões de Restinga do Parque Estadual de Itapuã em relação a outros estudos em matas de Restinga do RS, Brasil.

\begin{tabular}{|c|c|c|}
\hline & $\begin{array}{l}\text { Similaridade } \\
\qquad(\mathrm{J}, \%)\end{array}$ & $\begin{array}{c}\text { Diversidade } \\
\text { (H', nats) }\end{array}$ \\
\hline $\begin{array}{l}\text { Mata psamófila do Arroio Itapuã } \\
\text { (Leite } \text { et al. 2002) }\end{array}$ & 45,00 & - \\
\hline $\begin{array}{l}\text { Lagoa do Peixe (Dorneles \& } \\
\text { Waechter 2004) }\end{array}$ & 42,86 & 2,65 \\
\hline Quintão (Moraes \& Mondin 2001) & 40,48 & 2,13 \\
\hline Emboaba (Dillenburg et al. 1992) & 36,11 & 1,98 \\
\hline Reserva Biológica do Lami (Brum 1995) & 5) 30,43 & 2,71 \\
\hline Morro Grande (Waechter et al. 2000) & 29,51 & 2,41 \\
\hline $\begin{array}{l}\text { Rondinha Velha (M.G. Rossoni, dados } \\
\text { não publicados) }\end{array}$ & 27,14 & 3,08 \\
\hline
\end{tabular}

correlação alta com as espécies Casearia decandra $(-0,91)$, Chrysophyllum marginatum $(-0,91)$, Tabebuia pulcherrima (-0,90), Cecropia pachystachya $(0,64)$ e Psidium cattleyanum $(0,64)$. Já no Eixo II, correlações altas foram verificadas com Erythroxylum argentinum (0,98), Guapira opposita $(0,98)$, Syagrus romanzoffiana $(0,98)$, Solanum pseudoquina $(0,80)$, Cecropia pachystachya $(0,60)$, Coussapoa microcarpa $(0,60)$ e P Pidium cattleyanum $(0,60)$. Na Fig. 3b, a ordenação dos capões usando o IVI de cada espécie complementa a representação do gradiente evidenciado na Fig. 3a. O Eixo I teve correlação alta com as espécies Sebastiania serrata $(0,98)$, Eugenia uruguayensis $(-0,86)$, Ocotea pulchella $(-0,81)$, Chrysophyllum marginatum $(-0,66)$ e Tabebuia pulcherrima $(-0,60)$. O Eixo II apresentou altas correlações com Cecropia pachystachya $(0,93)$, Coussapoa microcarpa $(0,93)$, Psidium cattleyanum $(0,93)$, Syagrus romanzoffiana $(0,91)$ e Dodonaea viscosa $(0,90)$.

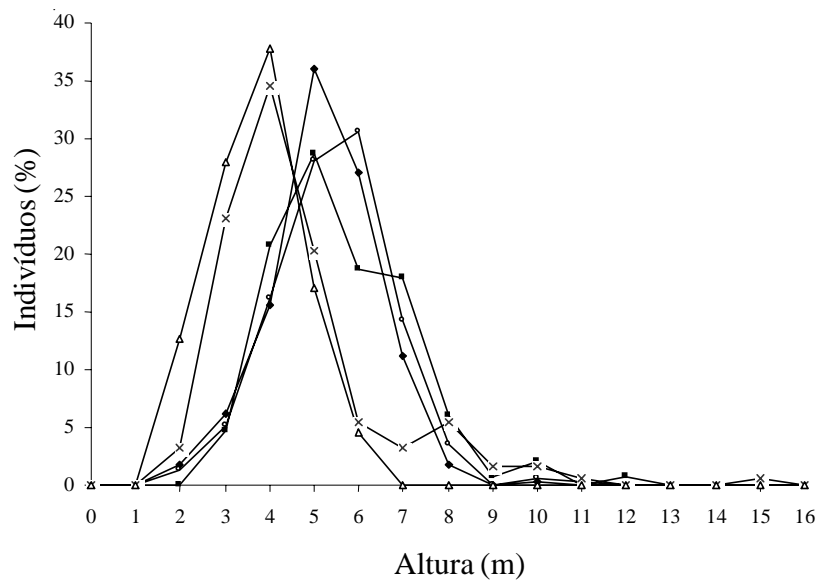

Figura 2. Distribuição da freqüência de alturas estimadas nos capões de Restinga do Parque Estadual de Itapuã, RS, Brasil. - - $=$ Tapera; - - - =Lagoinha; $-\mathrm{O}-=$ Coruja; $\longrightarrow x-=$ Puma; $-\triangle-=$ Capivara.

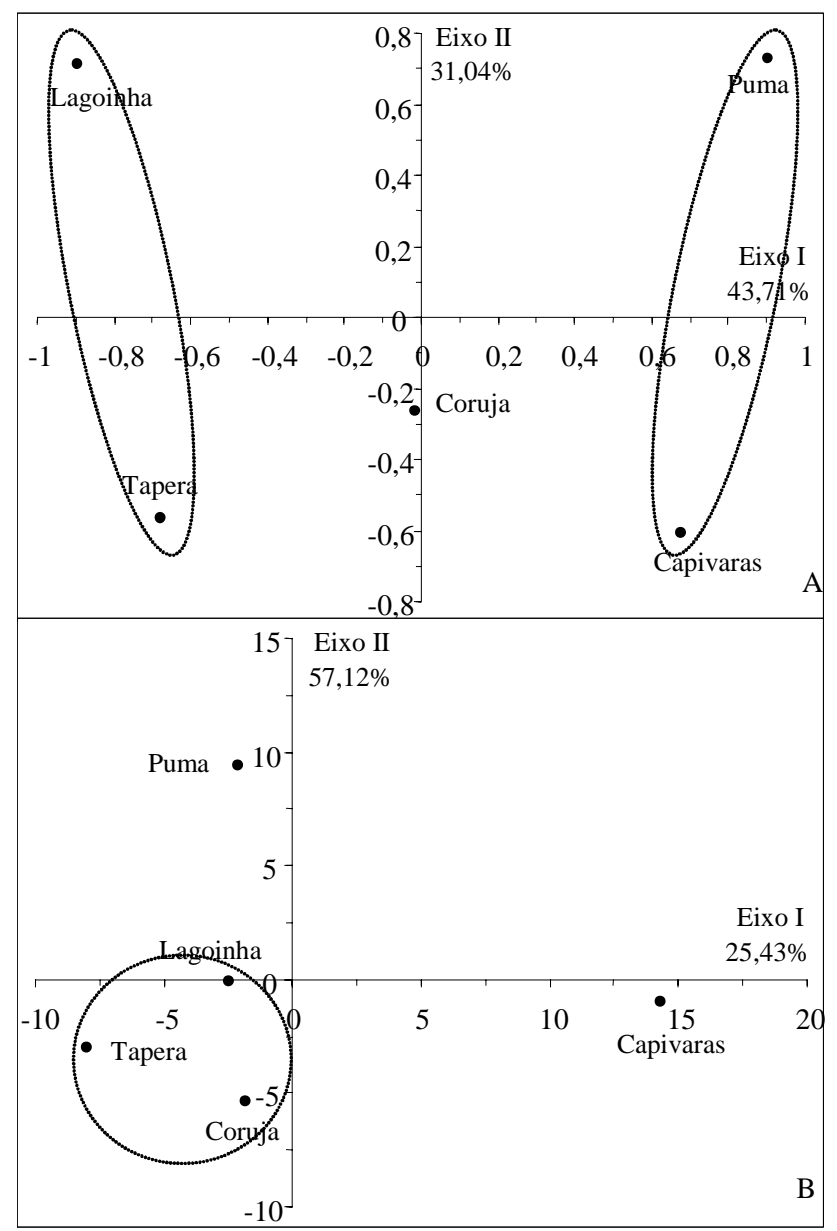

Figura 3. Análise de Coordenadas Principais dos capões de Restinga do Parque Estadual de Itapuã, RS, Brasil. A. Ordenação usando a composição florística. B. Ordenação usando o IVI de cada espécie. 


\section{Discussão}

As matas arenosas desenvolvem-se num ambiente extremo, onde os principais fatores que têm influência na flora são: escassez de nutrientes e água, mobilidade das dunas, excesso de calor e luz e a constância do vento (Rambo 1956; Dillenburg et al. 1992). Em vista destas condições, as plantas apresentam diversas características, conferindo um aspecto peculiar à vegetação, como por exemplo, baixo porte, com altura entre 6-12 m, muitos epífitos, diversidade de mirtáceas e a ocorrência de algumas espécies arbóreas características, entre elas as figueiras (Waechter 1985). As Restingas arbóreas da Praia de Fora, no Parque Estadual de Itapuã, também apresentaram estas características fisionômicas, onde foi constante o porte baixo. A constatação de somente um estrato superior e de um grupo de emergentes, acompanhados por um grande número de indivíduos de porte intermediário e baixo, que não formaram picos modais no diagrama de alturas, revela uma ausência de estratificação para essas matas de Restinga, o que também já foi encontrado em outros estudos (Dillenburg et al. 1992; L.P. Brum, dados não publicados; Dorneles \& Waechter 2004).

O caráter pioneiro dos ecossistemas de Restinga, citado anteriormente, faz com que poucas espécies tenham capacidade de colonizar e habitar estas áreas, influindo diretamente no número de famílias e espécies. O número de famílias encontrado nos capões de Restinga da Praia de Fora é baixo em relação a outros tipos florestais, porém não difere do encontrado em outros levantamentos de matas de Restinga arenosa, ficando em torno de 20 famílias (Dillenburg et al. 1992; L.P. Brum, dados não publicados; Waechter et al. 2000; Moraes \& Mondin 2001; Dorneles \& Waechter 2004).

A predominância de mirtáceas coincide com outros estudos em matas de Restinga (Dillenburg et al. 1992; Rossoni e Baptista 1994; 1995; Waechter et al. 2000; Moraes \& Mondin 2001; Dorneles \& Waechter 2004) realizados no sul do Brasil, visto que os membros da família encontram um ambiente propício para seu desenvolvimento (Reitz 1961). Segundo Araújo \& Lacerda (1987), as espécies da família Myrtaceae são muito comuns neste tipo de vegetação, sendo os gêneros Eugenia e Myrcia especialmente bem representados. Além desta família, Moraceae, Myrsinaceae, Cactaceae, Nyctaginaceae, Salicaceae e Urticaceae se fazem presentes nesta formação, com espécies que caracterizam a fisionomia da vegetação arbórea, destacando-se Ficus organensis, Myrsine guianensis, M. umbellata, Cereus hildmannianus, Guapira opposita, Casearia sylvetris e Coussapoa microcarpa.

A riqueza específica em matas de Restinga arenosa é relativamente baixa quando comparada com outras formações, podendo variar de 15 a 30 espécies aproximadamente (L.R. Dillenburg, dados não publicados; Dillenburg et al. 1992; L.P. Brum, dados não publicados; Waechter et al. 2000; Leite et al. 2002). O número de espécies total das Restingas do Parque Estadual de Itapuã está dentro deste limite, mas apresentou uma variação quando os capões foram analisados isoladamente.

As medidas de diversidade mais altas encontradas nos capões mais próximos ao morro granítico, matos da Tapera e da Lagoinha, reforçam a idéia de que esse local, revestido por Floresta Estacional Semidecidual, constitui-se numa fonte de propágulos. Espécies como Tabebuia pulcherrima, Casearia decandra e C. sylvestris, por exemplo, que são encontradas frequentemente em morros graníticos da região metropolitana de Porto Alegre (Knob 1975; Aguiar et al. 1986; Backes 1999), desaparecem gradualmente ao longo dos capões à medida que a distância do morro granítico aumenta. Esse gradiente é reforçado pela baixa riqueza no Mato das Capivaras, que é o mais distante do morro.

Além disso, a análise de agrupamento e de ordenação dos capões representou graficamente este gradiente, revelando que a distância entre os capões pode ser um fator que determina estas variações graduais na composição de espécies. Os capões mais próximos parecem formar grupos, tendo um maior número de espécies em comum. A formação desse gradiente na composição florística foi reforçada pela posição intermediária do Mato da Coruja entre dois grupos formados, conforme a análise de ordenação. Somando-se a isso, a ordenação através do IVI de cada espécie reforça o papel do fator distância na estrutura e composição dos capões, mostrando que os mais próximos apresentam maior semelhança na importância das espécies.

Estudos sobre Restinga apontam espécies do gênero Sebastiania entre as mais importantes, onde em muitos casos predomina no componente arbóreo (L.R. Dillenburg, dados não publicados; Dillenburg et al. 1992; L.P. Brum, dados não publicados; Leite et al. 2002). No presente estudo, a espécie $S$. serrata teve a maior importância em todos os capões de Restinga levantados, apresentando altos valores de densidade, frequiência e cobertura. Essa espécie apresenta uma síndrome de dispersão autocórica, além de frutificar ao longo do ano inteiro, o que pode ser uma vantagem 
no estabelecimento nessas áreas, juntamente com outras possíveis adaptações às condições limitantes impostas pelo ambiente (Rambo 1951; Klein 1975).

Ficus organensis também é uma das espécies fisionomicamente marcantes desta formação, mas geralmente apresenta baixa densidade e altos valores de cobertura. Na Praia de Fora, ela está presente entre as cinco espécies mais importantes nos capões, com exceção do Mato das Capivaras. Nesse capão e no Mato do Puma, a alta importância de D. viscosa demonstra um caráter de ocupação recente de espécies arbóreas, pois esta espécie é uma das primeiras a surgir na colonização de novas áreas em virtude de sua dispersão anemocórica e de adaptações a terrenos arenosos (Brack et al. 1998). Adicionalmente, no Mato do Puma, este alto valor de importância pode ser conseqüência da regeneração de áreas desmatadas. Quanto às demais espécies de maior importância nos cinco capões estudados, percebe-se uma coincidência com outros trabalhos realizados no sul do Brasil (L.R. Dillenburg, dados não publicados; Rossoni 1993; L.P. Brum, dados não publicados; Waechter et al. 2000; Moraes \& Mondin 2001).

A densidade total por hectare estimada para os cinco capões demonstra uma variação no sentido oesteleste, havendo uma redução de até $685 \mathrm{ind} / \mathrm{ha}$ (Mato da Lagoinha à Mato do Puma), atestando o caráter mais aberto dos capões mais orientais em relação ao morro granítico, fato que foi observado a campo. Isto também reforça a idéia de diferentes estágios de sucessão e/ou conservação.

Apesar da diferença entre os métodos de estudo, as medidas de diversidade estimadas para os capões do Parque Estadual de Itapuã são consoantes com o encontrado em outros estudos sobre Restingas (Dillenburg et al. 1992; L.P. Brum, dados não publicados; Waechter \& Jarenkow 1998; Waechter et al. 2000; Dorneles \& Waechter 2004). Estas comunidades vegetais enquadram-se num contexto de baixa diversidade devido à interação de fatores latitudinais, edafo-climáticos, temporais e históricos (Dillenburg et al. 1992). Segundo Wittaker (1972), as comunidades vegetais tendem a aumentar a sua diversidade específica no curso da sucessão.

Mesmo com diferenças nos critérios de inclusão utilizados, a comparação da composição florística com outros trabalhos mostrou que a área mais próxima, no Arroio Itapuã (Leite et al. 2002), teve maior similaridade e a mais distante, em Rondinha Velha (M.G. Rossoni, dados não publicados), foi menos similar. Neste último caso, a diferença na composição florística parece refletir a latitude em que a mata se encontra, tendo influência da Floresta Ombrófila Densa das escarpas da Serra Geral.

O índice de equabilidade (E) calculado representa uma média de $76 \%$ da máxima diversidade possível, considerando-se todos os capões, exceto o Mato das Capivaras, que apresentou maior índice de concentração (C). O reduzido número de espécies amostradas e a dominância de $S$. serrata contribuem para valores reduzidos de diversidade, situando-se entre os mais baixos já citados para comunidades arbóreas brasileiras (Waechter \& Jarenkow 1998).

Os resultados apresentados e discutidos indicam a existência de diferenças na composição florística e na estrutura da vegetação entre os capões de Restinga do Parque Estadual de Itapuã, que podem ser oriundos de diferentes estágios sucessionais, devido à ação antrópica ou causas naturais, bem como da distância de áreas fontes de sementes, como a Floresta Estacional Semidecidual. Certamente, um dos fatores que têm possibilitado a manutenção destas matas é o fato de estarem dentro de uma unidade de conservação, sobretudo dentro da área intangível do Parque (Rio Grande do Sul 1997). Considerando que as áreas de Restinga estão entre as mais impactadas dos ecossistemas brasileiros, são de grande importância estudos que retratem o estado de conservação destes ambientes.

\section{Agradecimentos}

Ao Prof. Sérgio Luiz de Carvalho Leite, pelos comentários e sugestões ao longo do trabalho; ao Botânico Marcos Sobral, pela identificação das mirtáceas; à Administração do Parque Estadual de Itapuã, por disponibilizar a infra-estrutura do parque; à CAPES, pela bolsa de Mestrado concedida ao primeiro autor.

\section{Referências bibliográficas}

Aguiar, L.W.; Martau, L.; Soares, Z.F.; Bueno, O.L.; Mariath, J.E. \& Klein, R.M. 1986. Estudo preliminar da flora e vegetação de morros graníticos da Região da Grande Porto Alegre, Rio Grande do Sul, Brasil. Iheringia, Série Botânica 34: 3-38.

APG II (Angiosperm Phylogeny Group). 2003. An update of the angiosperm phylogeny group classification of the orders and families of flowering plants: APGII. Botanical Journal of the Linnean Society 141: 399-436.

Araújo, D.S.D. 1992. Vegetation types of sandy coastal plains of tropical Brazil: a first approximation. Pp. 337-347. In: U. Seeliger (org.). Coastal Plant Communities of Latin America. New York, Academic Press. 
Araújo, D.S.D. \& Lacerda, L.D. 1987. A natureza das restingas. Ciência Hoje 6(33): 42-48.

Assumpção, J. \& Nascimento, M.T. 2000. Estrutura e composição florística de quatro formações vegetais de restinga no complexo lagunar Grussaí/Iquipari, São João da Barra, RJ, Brasil. Acta Botanica Brasílica 14(3): 301-315.

Backes, A. 1999. Ecologia da floresta do Morro do Coco, Viamão, RS. I - Flora e Vegetação. Pesquisas, Série Botânica 49: 5-30.

Brack, P.; Rodrigues, R.S.; Sobral, M. \& Leite, S.L.C. 1998. Árvores e arbustos na vegetação natural de Porto Alegre, Rio Grande do Sul, Brasil. Iheringia, Série Botânica 51(II): 139-166.

Costa, C.S.B.; Seeliger, U. \& Cordazzo, C.V. 1984. Aspectos da ecologia populacional do Panicum racemosum (Spreng) nas dunas costeiras do Rio Grande do Sul, Brasil. Pp. 395411. In: L.D. Lacerda; D.S.D. Araújo; R. Cerqueira \& B. Turcq (eds.). Restingas: origem, estrutura e processos. Niterói, CEUFF.

Dillenburg, L.R.; Waechter, J.L. \& Porto, M.L. 1992. Species composition and structure of a sandy coastal plain forest in northern Rio Grande do Sul, Brazil. Pp. 349-366. In: U. Seeliger (org.). Coastal Plant Communities of Latin America. New York, Academic Press.

Dorneles, L.P.P. \& Waechter, J.L. 2004. Estrutura do componente arbóreo da floresta arenosa de restinga do Parque Nacional da Lagoa do Peixe, Rio Grande do Sul. Hoehnea 31(1): 61-71.

Durigan, G. 2003. Métodos para análise de vegetação arbórea. Pp: 455-480. In: L. Cullen-Jr., R. Rudran \& C. ValladaresPadua (org.). Métodos de Estudos em Biologia da Conservação e Manejo da Vida Silvestre. Curitiba, Ed. da Universidade Federal do Paraná.

Falkenberg, D.B. 1999. Aspectos da flora e da vegetação secundária da restinga de Santa Catarina, Sul do Brasil. Insula 28: $1-30$.

Freire, M.S.B. 1990. Levantamento florístico do Parque Estadual das Dunas de Natal. Acta Botanica Brasilica 4: 41-59.

Gleasson, H.A. 1922. On the relation between species and area. Ecology 3(2): 158-162.

Kent, M. \& Coker, P. 1995. Vegetation description and analysis: a practical approach. Chichester, John Wiley.

Klein, R.M. 1975. Southern brazilian phytogeographic features and the probable influence of upper quaternary climatic changes in the floristic distribution. Boletim Paranaense de Geociências 33: 67-88.

Knob, A. 1975. Levantamento fitossociológico da formação-mata do Morro do Coco, Viamão, RS, Brasil. Iheringia, Série Botânica 23: 65-108.

Lamego, A.R. 1946. O homem e a Restinga. Rio de Janeiro, IBGE.

Leite, S.L.C.; Luz, M. \& Lange, O. 2002. Ecossistema terrestre: a mata ciliar. Pp. 57-65. In: O. Lange \& T. Guerra (orgs.). Análise ambiental da sub-bacia do Arroio Itapuã: Caderno para Educação Ambiental. Porto Alegre, Departamento de Ecologia/UFRGS.

Lindman, C.A.M. 1906. A vegetação no Rio Grande do Sul. Porto Alegre, Livraria Universal.
Moraes, D. \& Mondin, C.A. 2001. Florística e fitossociologia do estrato arbóreo e mata arenosa no balneário do Quintão, Palmares do Sul, Rio Grande do Sul. Pesquisas, Série Botânica 51: 87-100.

Mota, F.S. 1951. Estudos do clima do Estado do Rio Grande do Sul, segundo o sistema de W. Köppen. Revista Brasileira de Geografia 13(2): 275-284.

Mueller-Dombois, D. \& Ellenberg, H. 1974. Aims and methods of vegetation ecology. New York, John Wiley.

Pillar, V.D. 2001. MULTIV, Software for Multivariate Exploratory Analysis and Randomization Testing. Porto Alegre, Departamento de Ecologia, Uuniversidade Federal do Rio Grande do Sul.

Rambo, B. 1951. A imigração da selva higrófila no Rio Grande do Sul. Anais Botânicos do Herbário Barbosa Rodrigues 3(3): 55-91.

Rambo, B. 1954. História da flora do litoral riograndense. Sellowia 6: 113-172.

Rambo, B. 1956. A Fisionomia do Rio Grande do Sul. 2 ed., Porto Alegre, Livraria Selbach.

Reitz, R. 1961. A vegetação da zona marítima de Santa Catarina. Sellowia 13(13): 17-115.

Rio Grande do Sul, 1997. Plano de manejo Parque Estadual de Itapuã/RS. Porto Alegre, Secretaria da Agricultura e Abastecimento, Departamento de Recursos Naturais Renováveis.

Rossoni, M.G. \& Baptista, L.R.M. 1994/1995. Composição florística da mata de restinga, Balneário Rondinha Velha, Arroio do Sal, RS, Brasil. Pesquisas, Série Botânica 45: 115-131.

Sobral, M. 2003. A família Myrtaceae no Rio Grande do Sul. São Leopoldo, UNISINOS.

Streck, E.V.; Kämpf, N.; Dalmolin, R.S.D.; Klamt, E.; Nascimento, P.C. \& Schneider, P. 2002. Solos do Rio Grande do Sul. Porto Alegre, Ed. da Universidade Federal do Rio Grande do Sul.

Teixeira, M.B.; Coura-Neto, A.B.; Pastore, U. \& Rangel-Filho, A.L.R. 1986. Vegetação: As regiões fitoecológicas, sua natureza e seus recursos econômicos - Estudo fitogeográfico. Pp: 541-620. In: Levantamento de Recursos Naturais. v. 33, Rio de Janeiro, IBGE.

Waechter, J.L. 1985. Aspectos ecológicos da vegetação de restinga no Rio Grande do Sul, Brasil. Comunicações do Museu de Ciências da PUCRS, Série Botânica 33: 49-68.

Waechter, J.L. 1990. Comunidades vegetacionais das restingas do Rio Grande do Sul. Pp. 228-248. In: Anais do Simpósio de Ecossistemas da Costa Sul e Sudeste Brasileira. Águas de Lindóia 1990. São Paulo Publicação, ACIESP N. 71-3. v. 3.

Waechter, J. L. \& Jarenkow, J. A. 1998. Composição e estrutura do componente arbóreo nas matas turfosas do Taim, Rio Grande do Sul. Biotemas 11(1): 45-69.

Waechter, J.L.; Müller, S.C.; Breier, T.B. \& Venturi, S. 2000. Estrutura do componente arbóreo em uma floresta subtropical de planície costeira interna. Pp. 92-112. In: Anais do V Simpósio de Ecossistemas Brasileiros. Vitória 2000. Publicação ACIESP N. 109-III. v. 3. Espírito Santo.

Wittaker, R. H. 1972. Evolution and measurement of species diversity. Taxon 21(2/3): 213-251. 